http://www.jfas.info

\title{
INVESTIGATE OF WAVE ABSORPTION PERFORMANCE FOR OIL PALM FROND AND EMPTY FRUIT BUNCH AT 5.8 GHZ
}

E. Baharudin ${ }^{1,3,}{ }^{*}$, A. Ismail ${ }^{1,2}$, A. R. H. Alhawari ${ }^{1}$, E. S. Zainudin ${ }^{1}$, D. L. A. A. Majid ${ }^{1}$ and F. C. $\operatorname{Seman}^{3}$

${ }^{1}$ Faculty of Engineering, Universiti Putra Malaysia, 43400 Serdang, Selangor, Malaysia

${ }^{2}$ Institute of Advanced Technology, Universiti Putra Malaysia,43400 Serdang, Selangor, Malaysia

${ }^{3}$ Faculty of Electrical and Electronic Engineering, UniversitiTun Hussein Onn Malaysia, 86400 Parit Raja, Johor, Malaysia

Published online: 10 September 2017

\begin{abstract}
Low cost composites made from oil palm agricultural wastes showed much interest for absorber applications. In this work, dielectric properties of oil palm frond/PLA and empty fruit bunch/PLA which consists of 30\%wt, fiber and 70\%wt. polymer were determined using S-parameter Reflection Method and the microwave absorbers were design using CST at 5.8 GHz. The absorber thickness was varied to observe the reflection and transmission performance between 2 to $8 \mathrm{GHz}$. In addition, the Absorption Rate Figure of Merit and the absorber operating bandwidth were also analyzed. It is observed that both OPFPLA and EFBPLA able to absorb signal for about $20 \%$ per unit wavelength and have more than $50 \%$ absorption bandwidth.
\end{abstract}

Keywords: permittivity; biocomposite; microwave absorber; absorption rate.

Author Correspondence, e-mail:elfarizanis@gmail.com

doi: http://dx.doi.org/10.4314/jfas.v9i3s.27 


\section{INTRODUCTION}

Absorber is defining as a three-dimensional filtering device that does not transmit nor counter back the electromagnetic signal of a certain frequency band [1]. Hence, the necessity for the absorber to work in microwave [21] frequencies has become great concern especially in the application of military radar stealth for the prevention of electromagnetic energy [2]. Furthermore, the interest in microwave absorbers has become more profound especially in the issues of electromagnetic interference (EMI), radar signature reduction and radar absorbent materials (RAMs). The key requirements for microwave absorber are the ability to absorb or complement signals for a wide range of frequency band and they should be light in weight particularly for the reduction of aircraft payloads. Hence, it is important to understand the properties of material to meet the absorber requirements. The operating frequency is also a part of absorber requirements and by using the $5.8 \mathrm{GHz}$ of the industrial, scientific and medical (ISM) radio band, the absorber can be designed and performance can be observed.

Recently, much attention has been paid to environmental conservation issues especially the use of natural fibers biocomposites as a substitution to those hazardous and toxic materials which is hard to be disposed. Biocomposites have environmental advantages such as low density, high specific strength, enhanced energy recovery, $\mathrm{CO}_{2}$ neutrality after burning, easy processing, bio-degradability and low cost [3-4]. Hence, many studies have been made to realize the prospective of using agricultural waste such as rice husk, kenaf, sugarcane bagasse and pineapple leaf fiber as an alternative material for electromagnetic absorber [5-9].

Oil palm tree is a tropical palm plant, which can develop well within Malaysian environment. As a part of economic growth, oil palm trees plantation is seen as the most important agricultural crop in Malaysia [10]. With the development in oil palm industries, the percentage of agricultural wastes such as oil palm fronds and empty fruit bunch has expanded as well. By taking advantage of the agricultural wastes, therefore, this paper discusses the possibility of using oil palm fronds and empty fruit bunch [18] as an alternative material in the development of microwave absorbers at $5.8 \mathrm{GHz}$.

For any medium, the main characteristics are based on the electric permittivity, $\varepsilon$ and magnetic permeability, $\mu$ as shown in Fig. 1. Like an absorber, the main properties are the 
permittivity which is an extent of the material's effect on the electric field in the electromagnetic wave and the permeability is a measure of the material's effect on the magnetic component of the wave. When an electromagnetic field travels in the air and hits an absorber apart of the incident signal will be reflected, apart will be transmitted and apart will be absorbed. For a dielectric material, the complex form of permittivity is given by:

$\varepsilon^{*}=\varepsilon^{\prime}-j \varepsilon^{\prime \prime}$

where $\varepsilon^{\prime}$ is the dielectric constant or stored component and $\varepsilon^{\prime \prime}$ is loss factor or dissipative or lossy component. Both components are relative to free space permittivity $\varepsilon_{0}=8.85 \times 10^{-12}$ farads/meter.A material's levels of absorption can be determined by the ratio of lossy to stored components [11]. This is also known as material loss tangent which written mathematically as:

$\tan \delta=\frac{\varepsilon^{\prime \prime}}{\varepsilon^{\prime}}$

In order to know whether the material works effectively as an absorber is when the loss tangent is high. This means as the wave travels through the material, which results in the higher attenuation value, hence, more signal is being absorbed. Another important parameter of an absorber is the percentage of absorption rate (AR) and this is a frequency dependent parameter. When electric field of a specific frequency hits a surface of a medium, there will a reflected signal $S_{11}$ and transmitted signal $S_{21}$. Therefore, based on these conditions, the reflectance and transmittance factor can be denoted as the S parameter properties. Therefore, the absorption rate can be determined as [12]:

$A R(\omega)=1-\left|S_{11}\right|^{2}-\left|S_{21}\right|^{2}$

\section{METHODOLOGY}

In this research, agricultural wastes from oil palm trees such as oil palm fronds (OPF) and empty fruit bunches (EFB) are the main focus and they are used as fillers in the biocomposite. According to [10], OPF are wastes from oil palm plantation where they are always available due to the pruning process throughout the fruit harvesting. Meanwhile, EFB are obtained from the cooked and stripped off fresh fruits bunches. Table 1 shows the chemical composition for both, OPF and EFB. Biocomposite[19] is a combination of filler and matrix. In this research, OPF and EFB are set as fillers. Meanwhile, polylactic acid (PLA) is set as the matrix. PLA is 
a biogradable thermoplastic polyester that is manufactured by biotechnology processes from renewable resources [13]. PLA are polymers which is produced naturally from its monomer unit or by the process of fermentation, and then the polymerization procedure is a classical synthetic procedure [14]. Besides that, PLA is an eco-friendly polymer and has good mechanical properties such as high modulus, high strength and good clarity [13] which is a good option for an absorber matrix.

\subsection{Sample Preparation}

There are two types of samples. The first type of sample (OPFPLA) is prepared by mixing $30 \%$ wt. of OPF and 70\%wt. of PLA, and the second type is (EFBPLA) which is from 30\%wt. of EFB and 70\%wt. PLA. Before mixing procedure, the samples were prepared accordingly using the same techniques used in [15]. Then, the mixing process was continued by first calculating the composite final density to determine the weight of fiber and matrix to be mixed. Based on the internal mixer chamber, the allowed volume is given by $55 \mathrm{~cm}^{3}$. Hence, the sample mass is obtained by multiplying the volume with the material final density and $\mathrm{K}$ where $\mathrm{K}$ is a range between 0.7 and 0.8. The densities of OPFPLA and EFBPLA are 1.319 $\mathrm{g} / \mathrm{cm}^{3}$ and $1.315 \mathrm{~g} / \mathrm{cm}^{3}$ respectively. Then, the amount of fiber and PLA were mixed in an internal mixer with blade speed of $22 \mathrm{rpm}$ where the PLA pellets were gradually inserted in the mixing chamber until all melted at $180^{\circ} \mathrm{C}$. Next, the fibers were blend mix with the melted PLA and the whole process were done in 10 minutes. Later, the mixed materials are cooled and followed by the crushing process using crusher machine and finally they are ready for injection moulding process.

The sample's mould was designed based on the sample requirement of the S-Parameter Reflection Method where the samples were prepared in ring shaped figure as shown in Fig. 2(a). The nominal value for inner diameter (2r) is $3.05 \mathrm{~mm}$ and the outer diameter (2R) is 6.97 $\mathrm{mm}$. The ring sample height $(\mathrm{t})$ is $2.0 \mathrm{~mm}$. The injection moulding process were set according to material suitability where the process temperature was between $160^{\circ} \mathrm{C}$ to $180^{\circ} \mathrm{C}$, injection and cooling time were set to 3 seconds and 20 seconds respectively. The final ring samples are shown in Fig. 2(b). Two procedures involving the development of microwave absorbers using OPFPLA and EFBPLA materials are firstly is by determining the permittivity of OPFPLA and 
EFBPLA using the S-Parameter Reflection Method. Secondly, the microwave absorber is designed using CST.

\subsection{Permittivity Determination}

S-Parameter Reflection Method by Keycomm has the same concept of a typical coaxial line reflection method. This method measures the complex permittivity $\left(\varepsilon_{\mathrm{r}}\right)$ and complex permeability $\left(\mu_{\mathrm{r}}\right)$ at the same time. It uses the coaxial line as the feeder, which is fixed to conductive holder that holds the sample in a circular resonator. The other end of the coaxial line is connected to a vector network analyzer (VNA), where the reflected signal is analyzed and summarized using a computer based software. The calibration process between the VNA and the coaxial cable must be done first using open, short and $50 \Omega$ termination.

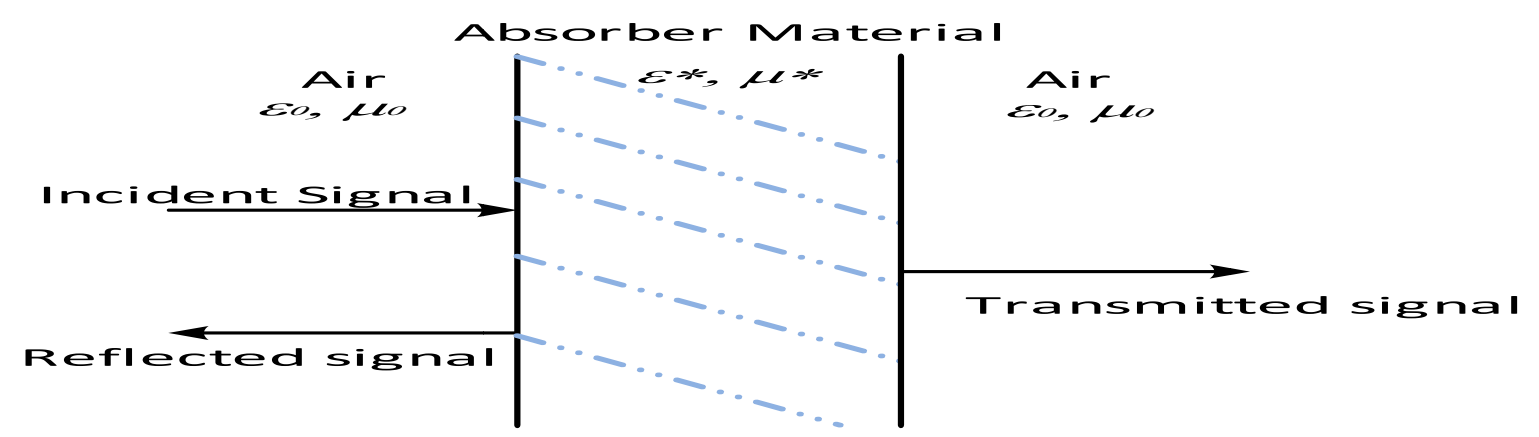

Fig.1. Absorber properties

Table 1. Chemical composition [16]

\begin{tabular}{ccc}
\hline Composition & OPF & EFB \\
\hline Cellulose (\%) & 42.2 & 47.9 \\
Hemicellulose \%) & 26.4 & 17.1 \\
Lignin (\%) & 22.3 & 24.9 \\
Extractives (\%) & 3.3 & 3.7
\end{tabular}

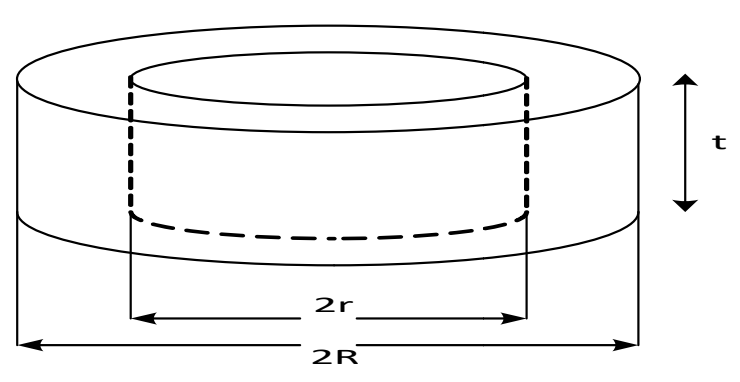

(a)

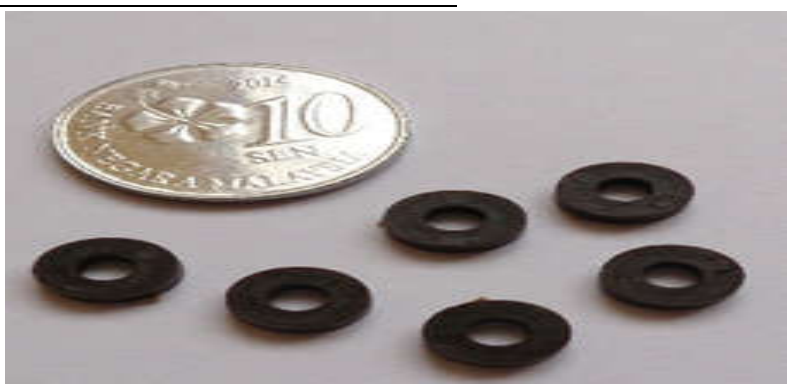

(b)

Fig.2. Samples preparation (a) samples dimensions(b) actual samples 
Then, the calibrated coaxial cable is fixed with the sample holder. At the termination of the sample holder, there will be either a piece of metal for shorting element or a resin that works as an open element. The open and short elements are used as the calibration tools for the sample holder. Once calibrated, the new sample is then inserted in the sample holder. Open and short fixtures are shown in Fig. 3. In S-Parameter Reflection Method, the calibration such as open and short play an important role to minimize the uncertainty errors. The selection of standard material such as air, Teflon and Rexolite 1422 help to minimize the uncertainties of the result [17]. The advantages of this technique are that the measurement can be done for a wide range of frequency and gives quite good results for the material dielectric properties.

\subsection{Absorber Simulation}

The absorbers were simulated in CST- Computer Simulation Technology software using two ports in unit cell boundary conditions over a frequency range of 2 to $8 \mathrm{GHz}$. The simulation of OPFPLA and EFBPLA microwave absorbers were simulated at $5.8 \mathrm{GHz}$ operating frequency. The dielectric constant and loss tangent of each material determined from the S-Parameter Reflection Method were set in the simulator as input parameters. The absorber has three dimensional parameters which are width, (w), length (l) and thickness (d). In the absorber design, $\mathrm{w}=1=2 \times \lambda_{\text {eff }}$ where $\lambda_{\text {eff }}$ the effective wavelength of the operating frequency which is $5.8 \mathrm{GHz}$. The thickness $\mathrm{d}$ is varied from $0.25 \lambda$ to $0.55 \lambda$, so that the optimum $\mathrm{d}$ is obtained. Optimum thickness is achieved when the best minimum reflectivity of the absorber is obtained.

\section{RESULTS AND DISCUSSION}

Fig. 4 shows the results of dielectric constants and loss tangents for OPFPLA and EFBPLA from 2 to $8 \mathrm{GHz}$ using the S-Parameter Reflection Method. It was observed that in Fig. 4(a) at the lower side of the frequency range, the dielectric constant of both OPFPLA and EFBPLA have higher value. As the frequency gets higher, the lower the dielectric constant gets. The average dielectric constant for OPFPLA and EFBPLA obtained were 2.622 and 2.503 respectively. As in Fig. 4(b), the loss tangents for both composites are observed to be fluctuating between 0.027 up to 0.07 . The average loss tangents determined were 0.045 and 
0.037 for OPFPLA and EFBPLA respectively. It is noticed that both composites have loss tangents more than 0.01 , which means they are considered as low loss material [5] and have good potential as an absorber. The results of the dielectric constant and the loss tangents as summarized in Table 2 are then set as the input parameter for the CST simulation.
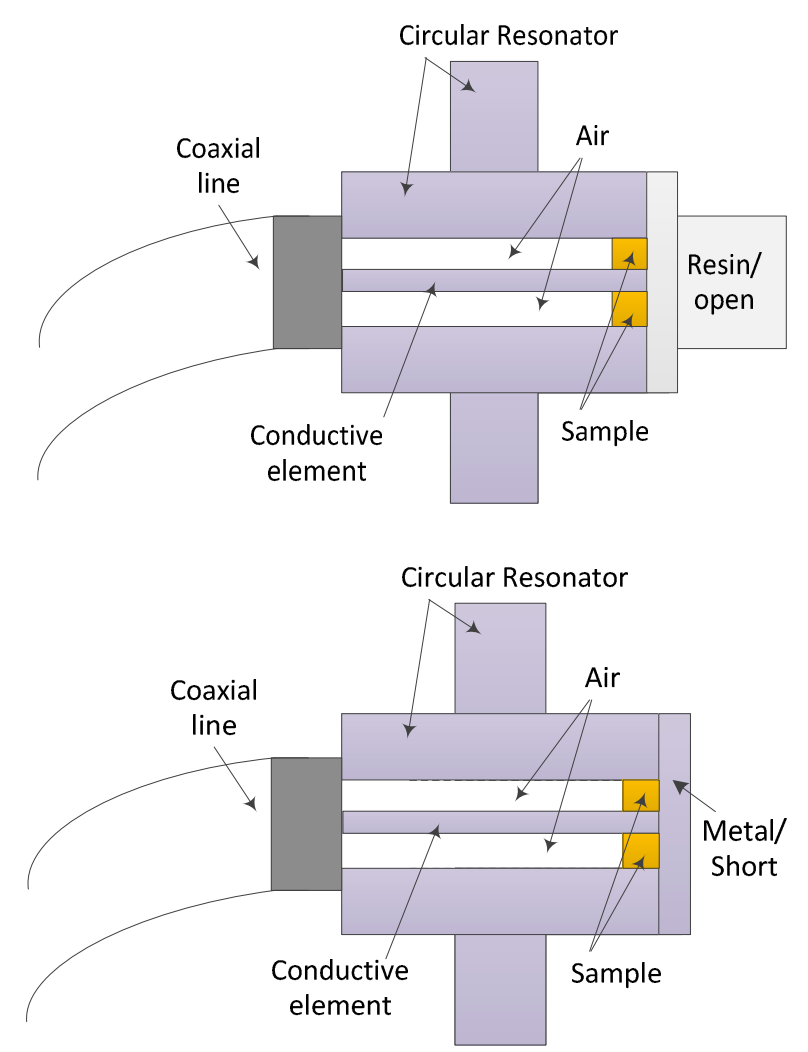

(a)

(b)

Fig.3. Calibration of S-Parameter Reflection Method (a) open (b) short

Two types of composite, OPFPLA and EFBPLA have been simulated at operating frequency of $5.8 \mathrm{GHz}$ and the signal reflection $\left(\mathrm{S}_{11}\right)$ and transmission $\left(\mathrm{S}_{21}\right)$ have been observed between 2 to $8 \mathrm{GHz}$. Fig. 5 shows the results of signal reflection, $\mathrm{S}_{11}$ for composite OPFPLA when the thickness $d$ is varied from $0.25 \lambda$ to $0.55 \lambda$. It was observed that when $d=0.25 \lambda$, the value of $\mathrm{S}_{11}$ was $-7.99 \mathrm{~dB}$ at frequency $5.8 \mathrm{GHz}$. As $d$ increases to $0.5 \lambda$ with every step of $0.05 \lambda$, it was noticed that the magnitude $\mathrm{S}_{11}$ also decreases and reaches to $-29.5 \mathrm{~dB}$. Another factor can be summarized for the signal reflection is that as the thickness of the absorber increases, the signal reflection band is shifted to lower side of the frequency and the magnitude also decreases at the resonating frequency.

On the other hand, Fig. 6 shows the result of the signal transmission, $\mathrm{S}_{21}$ for OPFPLA with the 
variation of thickness $\mathrm{d}$. When $\mathrm{d}=0.25 \lambda$, at the resonating frequency $5.8 \mathrm{GHz}$, the value of $\mathrm{S}_{21}$ was observed at magnitude of $-1.04 \mathrm{~dB}$. Then, it was noticed that the magnitude $\mathrm{S}_{21}$ increases to $-0.58 \mathrm{~dB}$ at $5.8 \mathrm{GHz}$ when $\mathrm{d}$ reaches $0.5 \lambda$. Hence, the signal transmission is proportionate with the increment of thickness d. To further investigate, the thickness (d) was now set to $0.55 \lambda$. It was noticed that the magnitude of $S_{11}$ has upturn to $-16.34 \mathrm{~dB}$ and $\mathrm{S}_{21}$ downturn to $-0.75 \mathrm{~dB}$ at the operating frequency of $5.8 \mathrm{GHz}$. When this happen, it can be predicted that the OPFPLA absorber has an optimum performance when the thickness is less than $0.5 \lambda$. Based on the same prediction, repeatedly, the same conditions were applied to EFBPLA composite.

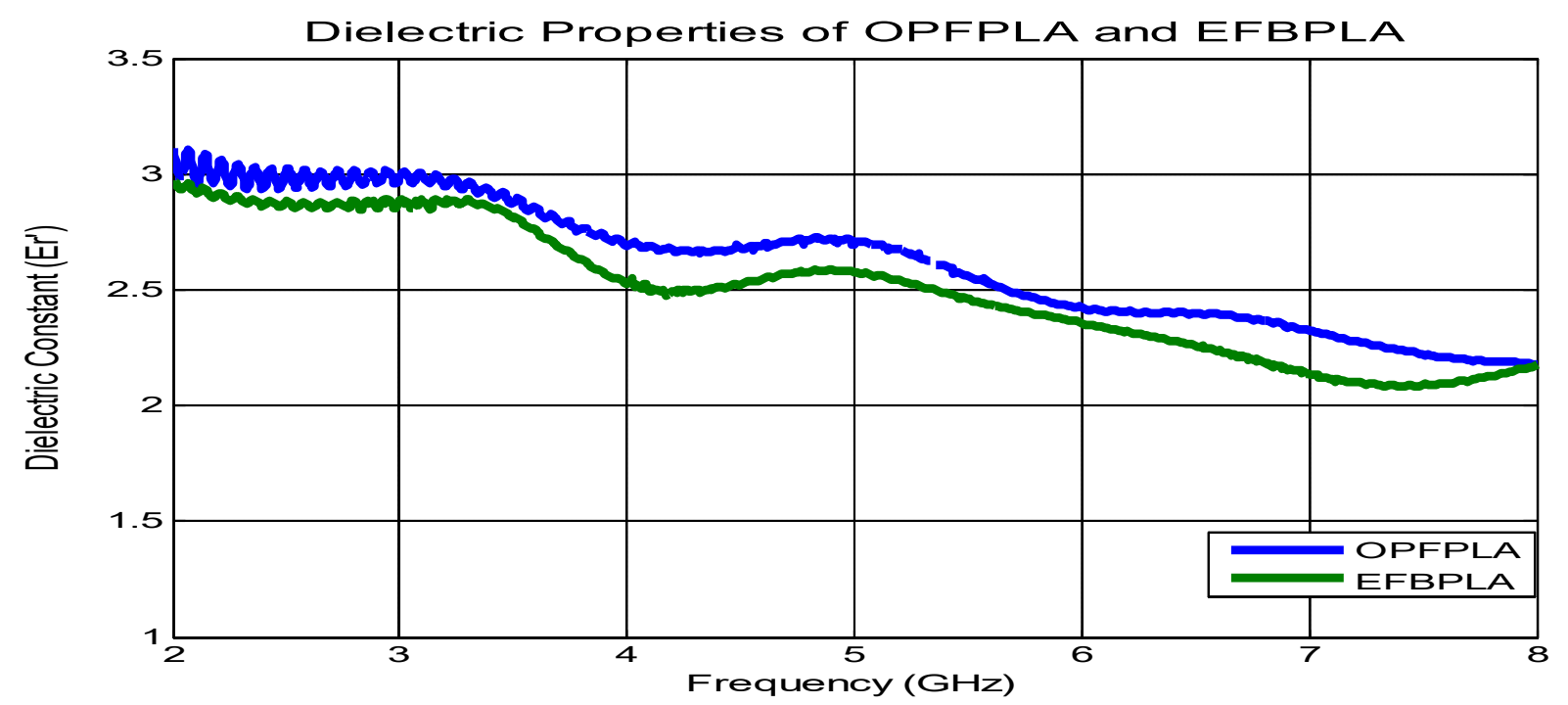

(a)

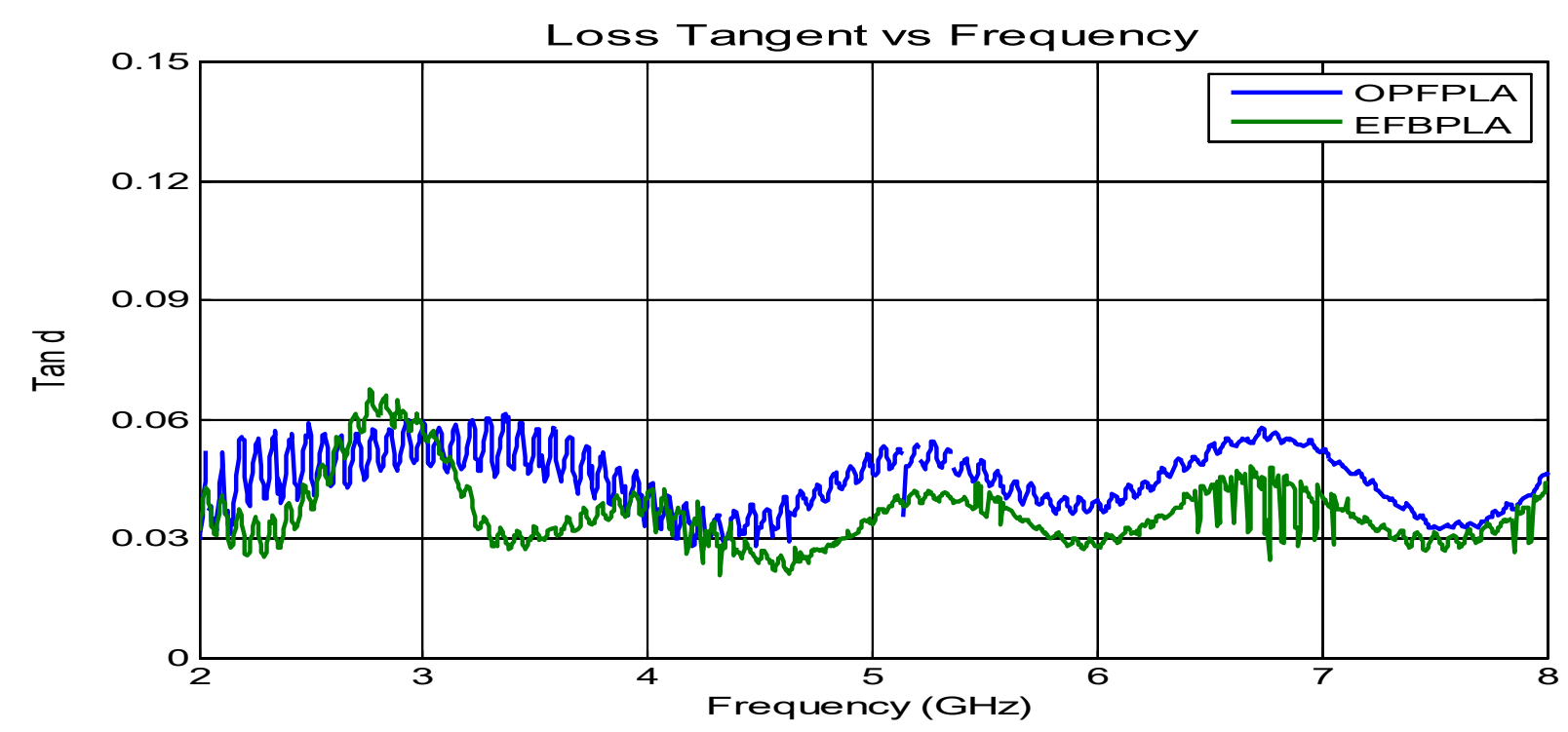


(b)

Fig.4. Dielectric properties of OPFPLA and EFBPLA (a) dielectric constant (b) loss tangent Fig. 7 shows the optimized thickness d of OPFPLA and EFBPLA absorbers, which give the optimum performance for $\mathrm{S}_{11}$ and $\mathrm{S}_{21}$ respectively at $5.8 \mathrm{GHz}$ operating frequency. It was noticed that both OPFPLA and EFBPLA have almost similar performances. Based on the results, the optimum thickness $d$ for OPFPLA and EFBPLA were $0.491 \lambda$ and $0.492 \lambda$ respectively which give the difference of one out of a thousand $\lambda$. For OPFPLA absorber, the optimum magnitude $\mathrm{S}_{11}$ and $\mathrm{S}_{21}$ were $-31.98 \mathrm{~dB}$ and $-0.56 \mathrm{~dB}$ respectively. On the other hand, as for EFBPLA absorber, the $\mathrm{S}_{11}$ and $\mathrm{S}_{21}$ have optimum magnitudes of $-34.19 \mathrm{~dB}$ and $-0.45 \mathrm{~dB}$ at $5.8 \mathrm{GHz}$ respectively. Comparatively, it was noticed that EFBPLA has slightly better performance in terms of reflectivity than OPFPLA with difference of about $2.2 \mathrm{~dB}$.

Then, to further investigate the performance of the absorber, the absorption rate (AR) as in Equation(3) was calculated based on the optimum results of $S_{11}$ and $S_{21}$. It was observed that the absorption rate for OPFPLA was $12.12 \%$ and EFBPLA has AR of $9.69 \%$. This shows that although EFBPLA has better minimum reflections than OPFPLA, but that does not signify the absorption is better. In addition, to further justify the absorber performance, the absorption rate Figure of Merit (FOM) is used to validate the results. The FOM is defined as the percentage of absorption rate (AR) at operating frequency divided by the absorber thickness per unit wavelength. This means that the AR is being normalized with the wavelength of the resonance frequency. It is important to see the effectiveness of the material in absorbing electromagnetic energy. Based on the optimum thickness results mentioned earlier, the calculated FOM for OPFPLA and EFBPLA were $24.67 \%$ and $19.68 \%$ respectively. This conforms that OPFPLA has better absorption than EFBPLA by $5 \%$.

Fig. 7 also shows another important parameter for an absorber which is the bandwidth. This is because it determines the capacity of data that can be transmitted. According to $5.8 \mathrm{GHz}$ ISM band, the allowable channel bandwidth is about $150 \mathrm{MHz}$ for an absorber, the bandwidth is determined by the intersection of the upper and lower cut-off frequencies of the $-10 \mathrm{~dB}$ line of the $S_{11}$ curve. Based on $S_{11}$ in Fig. 7, for OPFPLA, the upper and lower cut-off frequencies are observed at $7.58 \mathrm{GHz}$ and $4.11 \mathrm{GHz}$ respectively which gives $3.47 \mathrm{GHz}$ of bandwidth. As for 
EFBPLA, the upper and lower cut-off frequencies are $7.53 \mathrm{GHz}$ and $4.17 \mathrm{GHz}$ respectively which gives $3.36 \mathrm{GHz}$ of bandwidth. Both bandwidths cover more than 200 times of the 5.8 GHz ISM bandwidth. The bandwidth percentage is calculated when the obtained bandwidth is divided by the operating frequency and this is summarized in Table 3, where both OPFPLA and EFBPLA absorbers cover more than $50 \%$ with respect of the operating frequency.

Table 2. Average dielectric properties over 2 to $8 \mathrm{GHz}$

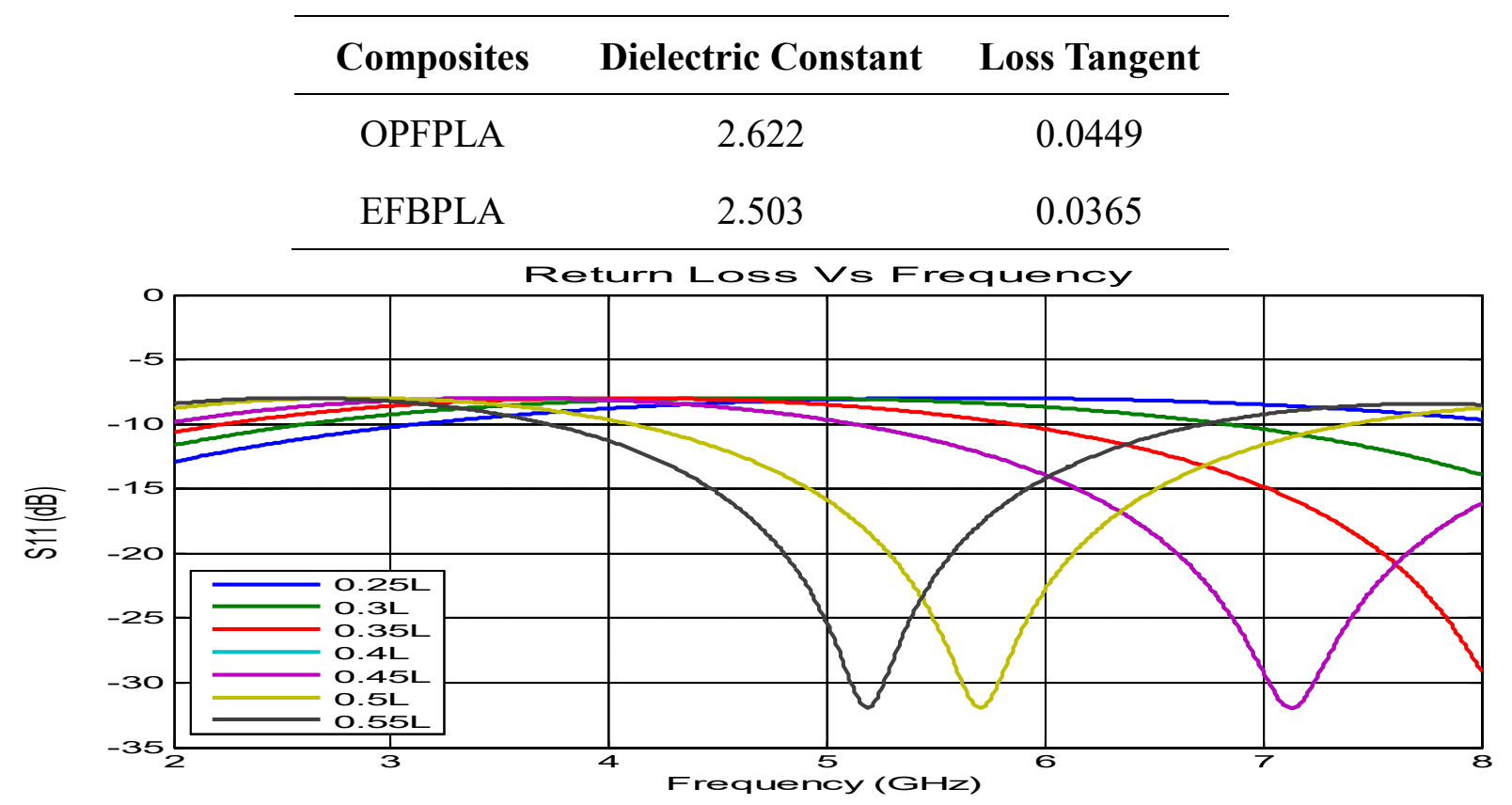

Fig.5. Signal reflection with thickness variation $(L=\lambda)$

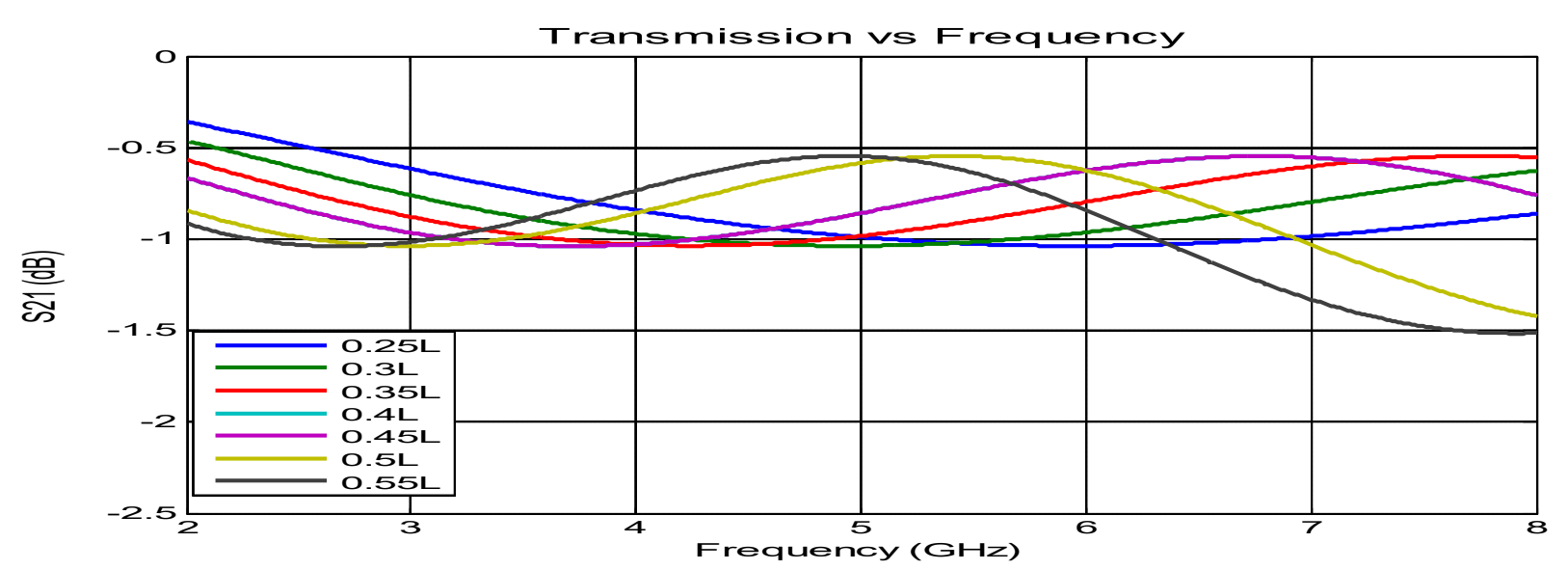

Fig.6. Signal transmission with thickness variation $(L=\lambda)$ 


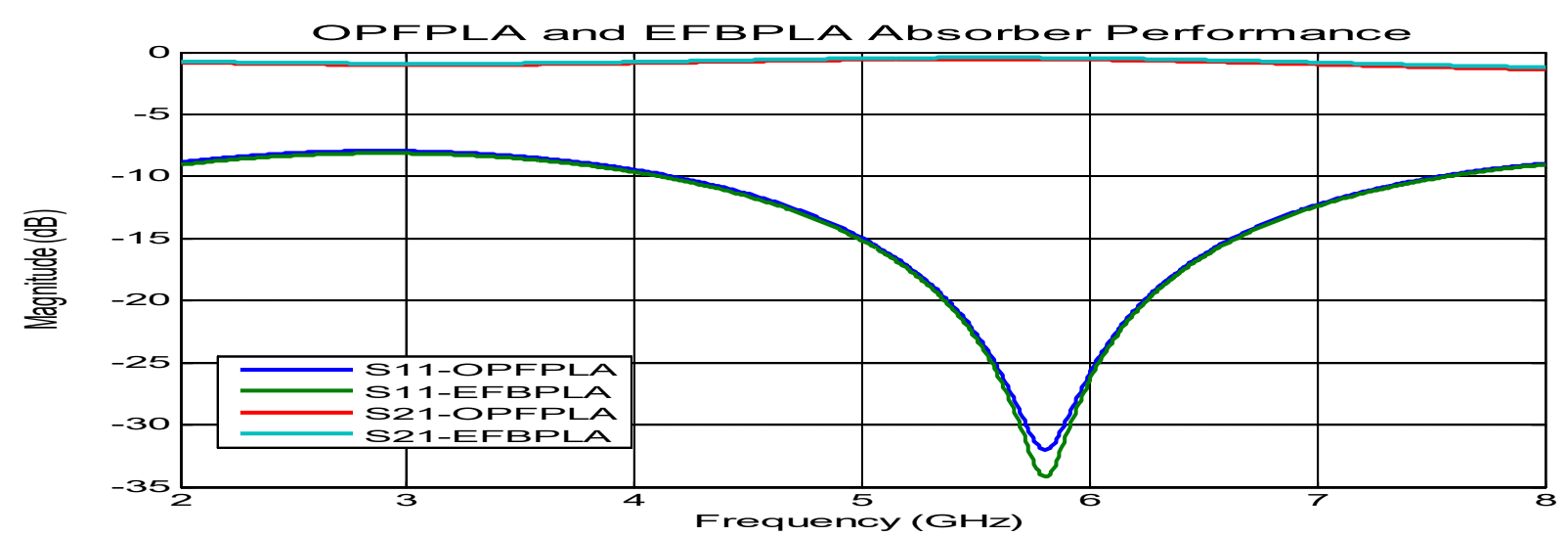

Fig.7. Optimizes performance for OPFPLA and EFBPLA

Table 3. Comparison of FOM and bandwidth for OPFPLA and EFBPLA

\begin{tabular}{ccc}
\hline Composites & Absorption Rate FOM (\%) & $\begin{array}{c}\text { Bandwidth } \\
(\%)\end{array}$ \\
\hline OPFPLA & 24.67 & 59.73 \\
EFBPLA & 19.68 & 58.04 \\
\hline
\end{tabular}

\section{CONCLUSION}

The work here demonstrates and justifies the absorber performance and effectiveness based on the agricultural waste biocomposites from oil palm tree, namely OPFPLA and EFBPLA at $5.8 \mathrm{GHz}$ operating frequency. The biocomposites[20] are found to have low loss material properties and able to show good performance in terms of signal reflection and transmission. Furthermore, both OPFPLA and EFBPLA have good absorption rate per wavelength and able to absorb signal for a wide range of frequency.

\section{REFERENCES}

[1]Kundu D, Mohan A, Chakrabarty A.single layer wideband microwave absorber using array of crossed dipoles.IEEE Antennas and Wireless Propagation Letters, 2016, 15:1589-1592

[2] Wang T, Wang P, Wang Y, Qiao L. A broadband far-field microwave absorber with a sandwich structure. Materials and Design, 2016, 95:486-489

[3] Bravo A, Toubal L, Koffi D, Erchiqui F. Development of novel green and biocomposite materials: Tensile and flexural properties and damage analysis using acoustic emission. 
Materials and Design, 2015, 66:16-28

[4] Faruk O, Bledzki A K, Fink H, Sain M.Biocomposites reinforced with natural fibers: 2000-2010. Progress in Polymer Science, 2012, 37(11):1552-1596

[5] Baharudin E, Ismail A, Alhawari A R, Zainudin E S, Majid D L, Che S F. Investigation on the dielectric properties of pulverized oil palm frond and pineapple leaf fiber for X-band microwave absorber application. Advanced Materials Research, 2014, 893:488-491

[6] Farhany Z S, Malek F, Nornikman H, Affendi N M, Mohamed L, Saudin N, Ali A A. Potential of dried banana leaves for pyramidal microwave absorber design. In IEEE Symposium on Wireless Technology and Applications, 2012, pp. 60-65

[7] Liyana Z, Malek F, Nornikman H, Affendi N M, Mohamed L, Saudin N, Ali A A. Investigation of sugar cane bagasse as alternative material for pyramidal microwave absorber design. In IEEE Symposium on Wireless Technology and Applications, 2012, pp. 66-70

[8] Noordin I R, Idris H A, Taib M N, Sharif J M, Rosli A D, Zanal A, Abdullah A T. Investigation of oil palm ash microwave absorber for broadband application. In IEEE 8th International Colloquium on Signal Processing and its Applications, 2012, pp. 232-235

[9] Zawawi N A, Ismail A, Abdan K, Mahdi M A. From waste to electronics: printed circuit boards using renewable resources of oil palm empty fruit bunch. Advanced Materials Research, 2012, 567:263-266

[10] Awalludin M F, Sulaiman O, Hashim R, Nadhari W N. An overview of the oil palm industry in Malaysia and its waste utilization through thermochemical conversion, specifically via liquefaction. Renewable and Sustainable Energy Reviews, 2015, 50:1469-1484

[11] Vinoy K. J., Jha R. M.Radar absorbing materials: From theory to design and characterization. Dordrecht: KluwerAcademic Publishers, 1996

[12] Rufangura P, Sabah C. Wide-band polarization independent perfect metamaterial absorber based on concentric rings topology for solar cells application.Journal of Alloys and Compounds, 2016, 80:473-479

[13] Saeidlou S, Huneault M A, Li H, Park C B. Poly(lactic acid) crystallization. Progress in Polymer Science, 2012, 37(12):1657-1677

[14] Bogoeva-Gaceva G, Avella M, Malinconico M, Buzarovska A, Grozdanov A, Gentile G, 
Errico ME. Natural fiber eco-composites. Polymer Composites, 2007, 28(1):98-107

[15] Baharudin E, Ismail A, Alhawari A R, Zainudin E S, Majid D A, Seman F C, Khamis N H. Determination of pulverized material permittivity for microwave absorber application. In IEEE Regional Symposium on Micro and Nanoelectronics, 2013 2013, pp. 85-88.

[16] Mohanty A. K., Misra M., Drzal L. T. Natural fibers, biopolymers and biocomposite. Florida: CRC Press, 2005

[17] Zawawi N A, Ismail A, Khalina A, Mahdi M A. Investigation on the microwave properties of kenaf and rice-husk fiber reinforced PLA composite utilizing one-port coaxial transmission line reflection method. Key Engineering Materials, 2011, 471:868-873

[18] Muhammad I A, Mohd S M R, Shahril N M S, Mazlan M, ZairiI R, Mohd H M A.Preliminary study of microwave irradiation towards oil palm empty fruit bunchesbiomass.Journal of Tropical Resources and Sustainable Science,2016, 8(1):133-137

[19] Siti A I, Mazlan M, Sitti F M R, Mohd H M A, Aziz M S A, Zairi I R. Biocomposite material to enhance heat transfer of wood (shorea faguetiana and palaquim sp) for green building in Malaysia.ARPN Journal of Engineering and Applied Sciences, 2015, 10(1):301-312

[20] Nik H A Nik R, Mazlan M, Marinah M, Mohd H M A, Aziz M S A, Zairi I R. Effect of biocomposite materials to enhance durability of selected wood species (intsia palembanica miq, neobalanocarpus heimii, shorea plagata) in Malaysia.ARPN Journal of Engineering and Applied Sciences, 2015, 10(1):313-320

[21] RizmanZ I, Jusoff K, Rais S S, Bakar H H, Nair G K S, Ho Y K. Microwave signal propagation on oil palm trees: Measurements and analysis. International Journal on Smart Sensing and Intelligent Systems,2011, 4(3):388-401 


\section{How to cite this article:}

Baharudin E, Ismail A, Alhawari A R H, Zainudin E S, Majid D L A A, Seman F C. Investigate of wave absorption performance for oil palm frond and empty fruit bunch at 5.8 ghz. J. Fundam. Appl. Sci., 2017, 9(3S), 335-348. 